\title{
Higgs boson measurements at Future Circular Colliders
}

\author{
Sylvie Braibant ${ }^{a \dagger, * *}$ \\ ${ }^{a}$ Physics and Astronomy Department, Bologna University, \\ Viale B. Pichat 6/2, Bologna, Italy \\ E-mail: sylvie.braibant@cern.ch
}

Precision measurements and searches for new phenomena in the Higgs sector are amongst the most important goals in particle physics. Experiments at the Future Circular Colliders (FCC) are ideal to study these questions. Electron-positron collisions (FCC-ee) up to an energy of $365 \mathrm{GeV}$ will allow to measure with a great precision the Higgs boson couplings, mass, total width, but also to search for exotic and invisible Higgs boson decays. Very high energy proton-proton collisions (up to $100 \mathrm{TeV}$ ) provided by the subsequent FCC-hh will allow a measurement of the Higgs boson self-coupling. There is a remarkable complementarity of the FCC-ee and FCC-hh colliders, which in combination offer the best possible overall study of the Higgs boson properties.

*** The European Physical Society Conference on High Energy Physics (EPS-HEP2021), ***

*** 26-30 July $2021 * * *$

*** Online conference, jointly organized by Universität Hamburg and the research center DESY ***

\footnotetext{
*Speaker

${ }^{\dagger}$ On behalf of all the FCC-ee and FCC-hh collaborators
} 


\section{Introduction}

At the High-Luminosity HL-LHC [1], the Higgs boson couplings to fermions and bosons are expected to be measured with a precision of the order of 5-10\% [2]. In order to significantly improve these measurements, new accelerator facilities are needed. The Future Circular Collider (FCC) [3] at CERN is a design study for a post-LHC collider and will be installed in a tunnel with a circumference of $97.5 \mathrm{~km}$. This project integrates a circular leptonic collider FCC-ee [4] in a first stage followed in a second stage by a proton-proton collider FCC-hh [5].

Advantages of circular lepton colliders are that they can achieve high luminosities and that they can later be replaced by very high energy pp colliders. The sequential implementation of a lepton and a hadron collider will therefore maximise the physics reach and covers more than 50 years of exploratory physics starting in the late 2030's.

The data taking program of the FCC-ee collider foresees to deliver $5 \mathrm{ab}^{-1}$ of integrated luminosity in 3 years of operation at $\sqrt{s}=240 \mathrm{GeV}$. The FCC-ee collider will also run at the $\mathrm{Z}$ peak and at the WW production threshold. Finally, the FCC-ee will be operated at the $t \bar{t}$ production threshold with an expected integrated luminosity of $1.5 \mathrm{ab}^{-1}$ delivered in 5 years. The data collected at these various center-of-mass energies make this machine the only one capable to provide extremely high precision measurements of all the electroweak sector.

The hadronic collider FCC-hh collider will instead be operated at $\sqrt{s}=100 \mathrm{TeV}$ and is expected to deliver data with an integrated luminosity of $30 \mathrm{ab}^{-1}$ in 25 years; in particular, it will allow the measurement of the Higgs boson self-coupling.

\section{Higgs boson measurements}

At FCC-ee, the Higgstrahlung process is the dominant production mode with a maximal cross section of about $200 \mathrm{fb}$ at $\sqrt{s}=240 \mathrm{GeV}$; in this process, the Higgs boson is produced together with a real $\mathrm{Z}$ boson, $e^{+} e^{-} \rightarrow H Z^{*} \rightarrow H Z$. The $\mathrm{WW}$ fusion process, $e^{+} e^{-} \rightarrow H v_{e} \overline{v_{e}}$ becomes relevant at $\sqrt{s}=365 \mathrm{GeV}$. The corresponding Feynman diagrams are shown in figure 1 .
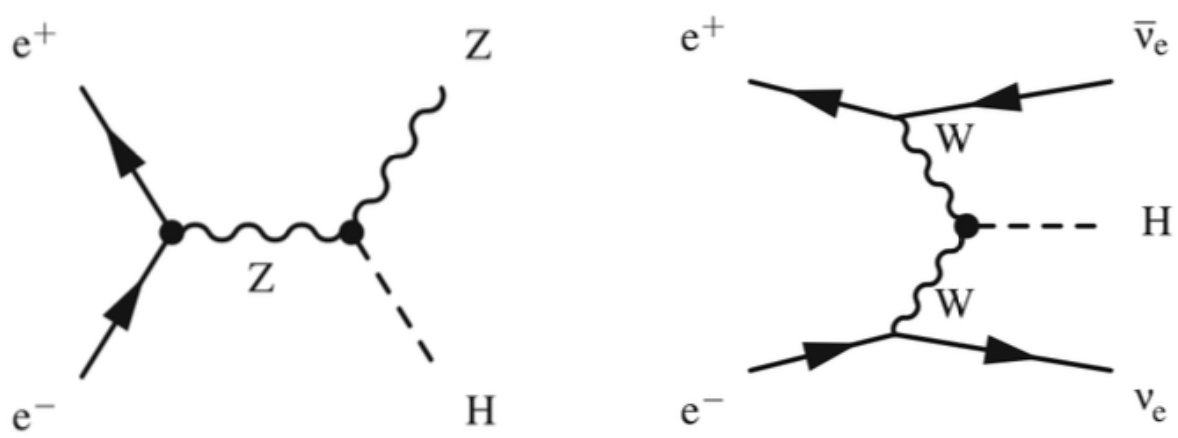

Figure 1: Feynman diagrams for Higgs boson production through the Higgsstrahlung (left) and the WW fusion (right) processes.

With $5 \mathrm{ab}^{-1}$ of data collected at $\sqrt{s}=240 \mathrm{GeV}$, FCC-ee will produce about 1 million HZ events. Another $180000 \mathrm{WW} \rightarrow \mathrm{H}$ are expected with $1.5 \mathrm{ab}^{-1}$ of data collected at $\sqrt{s}=365 \mathrm{GeV}$. 
In the Higgsstrahlung process, the Higgs boson coupling measurements can be performed independently of the Higgs boson decays: events are tagged with one identified $\mathrm{Z}$ boson and the mass recoiling against the $\mathrm{Z}$ decay products peaks around the Higgs boson mass.

This recoil mass method allows to measure the inclusive cross section of the $\mathrm{ZH}$ process directly, without making any assumption about the Higgs boson branching ratios. This possibility is unique to lepton colliders as it relies on the precise knowledge of the total initial energy of the event.

The recoil mass distribution obtained with a DELPHES simulation [7] of the IDEA detector concept [8] is shown in figure 2 for an integrated luminosity of $5 \mathrm{ab}^{-1}$. The signal events are $e^{+} e^{-} \rightarrow H Z^{*} \rightarrow H Z, Z \rightarrow \mu^{+} \mu^{-}$. The distributions arising from various background samples are also shown [9].
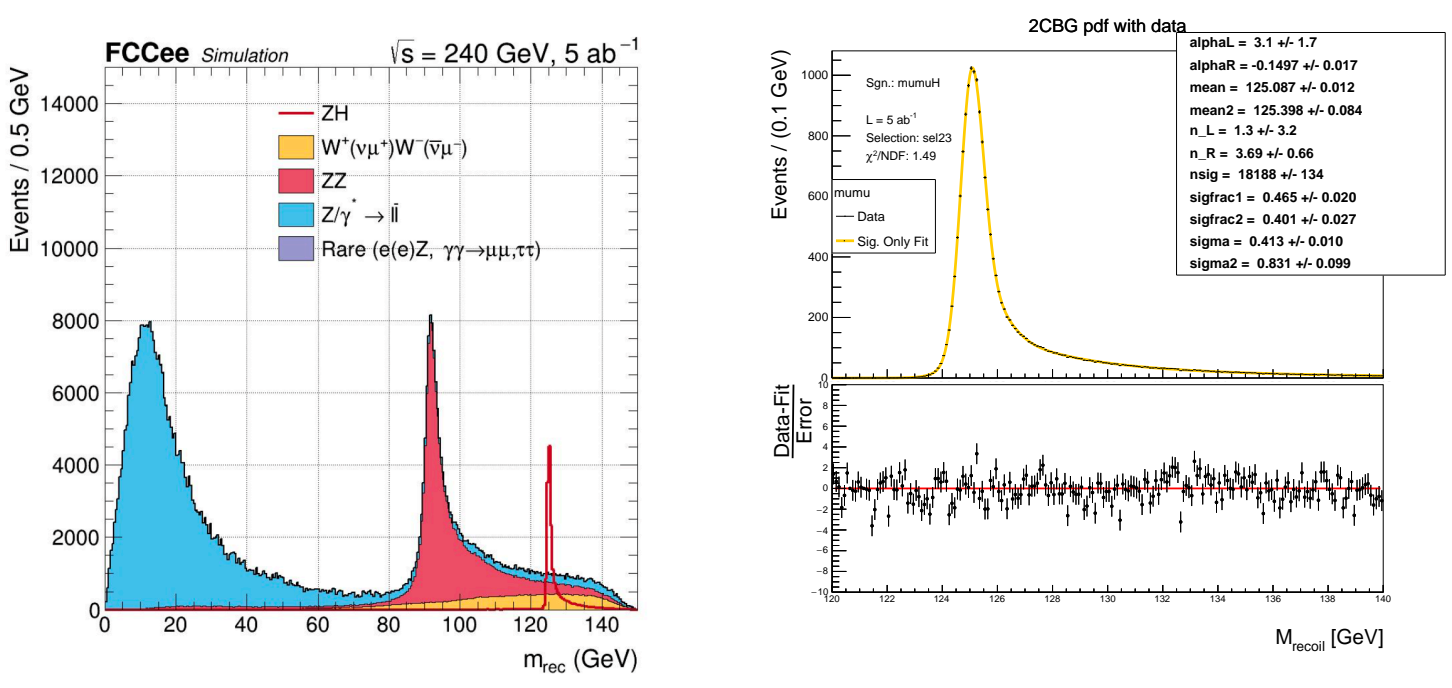

Figure 2: Recoil mass distributions for signal events $\left(e^{+} e^{-} \rightarrow H Z^{*} \rightarrow H Z, Z \rightarrow \mu^{+} \mu^{-}\right)$and for various background events (left) and recoil mass distributions for signal events in the region around $\mathrm{m}_{H}$, fitted with 2 Crystal-Ball plus a Gaussian (right) [9].

In combination with measurements of exclusive Higgs decay cross sections, the direct measurement of the inclusive cross section of the $\mathrm{ZH}$ process allows to extract the total width $\Gamma_{H}$. Combining the measurements obtained at $\sqrt{s}=240 \mathrm{GeV}$ with the ones at $365 \mathrm{GeV}$, one obtains a precision on the width of the Higgs boson, $\delta \Gamma_{H} / \Gamma_{H}$, of $1.1 \%$ [6].

By using the measured $\mathrm{HZ}$ cross section and the total width one can then perform absolute measurements of the couplings of the Higgs bosons to all the Standard Model (SM) particles in a model independent way. The relative precisions on the Higgs couplings, $\delta g_{H} / g_{H}$, obtained at FCC-ee combining the measurements with HL-LHC are shown in Table 1. Several Higgs couplings will therefore be measured at FCC-ee with a sub-percent precision. This represents roughly an order of magnitude improvement compared to what will be achievable at HL-LHC [6].

The FCC-ee will also allow to search for very rare events that could arise from new physics beyond the SM. For example Higgs boson to invisible decays are predicted by the Higgs-portal model of Dark Matter. This uses ZH events in which the Z boson decays to a pair of leptons or to a pair of $b$ quarks while the Higgs boson decays invisibly with an assumed branching ratio of $100 \%$. 


\begin{tabular}{lcc}
\hline Coupling & $\begin{array}{c}\text { FCC-ee 240 GeV } \\
\text { +HL-LHC } \\
\text { (in \%) }\end{array}$ & $\begin{array}{c}\text { +FCC-ee at 365 GeV } \\
\text { +HL-LHC } \\
\text { (in \%) }\end{array}$ \\
\hline$\delta g_{H W W}$ & 0.88 & 0.41 \\
$\delta g_{H Z Z}$ & 0.20 & 0.17 \\
$\delta g_{H g}$ & 1.2 & 0.9 \\
$\delta g_{H \gamma \gamma}$ & 1.3 & 1.3 \\
$\delta g_{H c c}$ & 1.5 & 1.3 \\
$\delta g_{H t t}$ & 3.1 & 3.1 \\
$\delta g_{H b b}$ & 1.0 & 0.64 \\
$\delta g_{H \mu \mu}$ & 4.0 & 3.9 \\
$\delta g_{H \tau \tau}$ & 0.94 & 0.66 \\
\hline
\end{tabular}

Table 1: Relative precision (in \%) on the Higgs couplings, $\delta g_{H} / g_{H}$, to SM particles that can be obtained at FCC-ee combining the measurements at HL-LHC. In the third column are reported the values obtained combining also the data taken with FCC-ee at $\sqrt{s}=365 \mathrm{GeV}$ [6].

The $95 \%$ confidence level (CL) upper limit on the branching ratio to invisible decays obtained with the data collected at $\sqrt{s}=240 \mathrm{GeV}$ is $0.22 \%$. Combining this measurement with the one at $\sqrt{s}=$ $365 \mathrm{GeV}$, one obtains $\mathrm{BR}_{i n v}<0.19 \%$ at the $95 \% \mathrm{CL}$ [6]. This result obtainable at FCC-ee improves by an order of magnitude the limit obtainable at HL-LHC. For the Higgs exotic decays, i.e. decays to final states that cannot be tagged as SM decays, the 95\% CL upper limit on the branching ratio is $\mathrm{BR}_{\text {unt }}<1.0 \%[6]$.
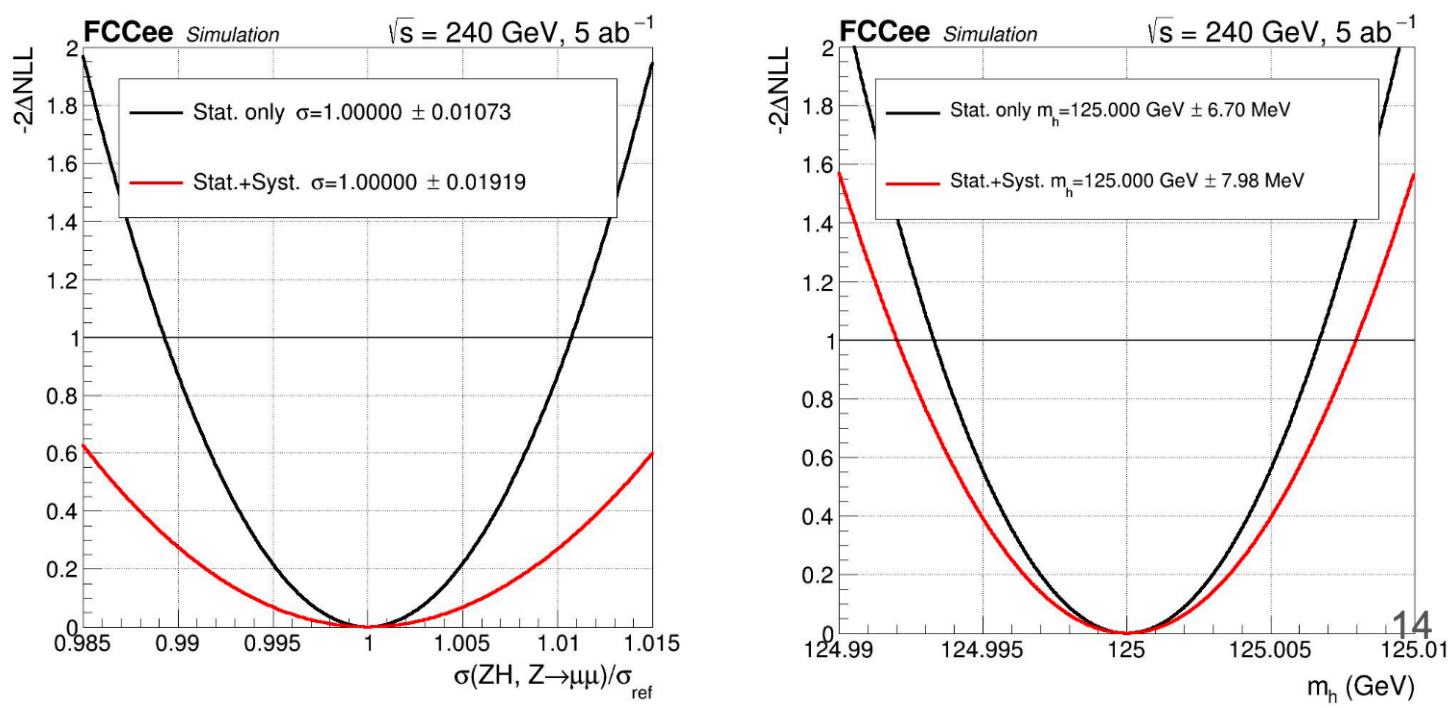

Figure 3: Negative log-Likelihood scans as a function of the signal strength $\mu$ and of the Higgs mass $\mathrm{m}_{H}$.

In order to improve the precision on the Higgs boson mass measurement, recent efforts are being invested in the recoil mass method to optimise and tune signal and background parameterisations but also to include systematic uncertainties for instance due to the beam energy spread (BEH) and initial state radiation (ISR). Negative log-Likelihood scans as a function of the signal strength $\mu$ 
and of the Higgs mass $\mathrm{m}_{H}$ are shown in figure 3. These preliminary results including systematic uncertainties indicate that a precision of about $1.9 \%$ on the signal strength $\mu$ and a precision of 8 $\mathrm{MeV}$ on the Higgs boson mass $\mathrm{m}_{H}$ are attainable at the FCC-ee [9].

\section{Higgs boson self-coupling measurement}

The precise measurement of the Higgs tri-linear self-coupling $\lambda_{3}$ must be a top priority at future high-energy collider experiments since it can constrain the shape of the Higgs potential. The circular $100 \mathrm{TeV}$ proton-proton collider FCC-hh would provide the most precise measurement of this crucial quantity. At $\sqrt{s}=100 \mathrm{TeV}$, the dominant $\mathrm{HH}$ production mode is the gluon fusion ( $\mathrm{ggHH}$ ) with a production cross section of $1224 \pm 5.6 \mathrm{fb}$ [10], about 40 times larger than the corresponding cross section at $\sqrt{s}=14 \mathrm{TeV}$. The di-Higgs production processes are shown in figure 4; these two diagrams negatively interfere hence yielding a small cross section.

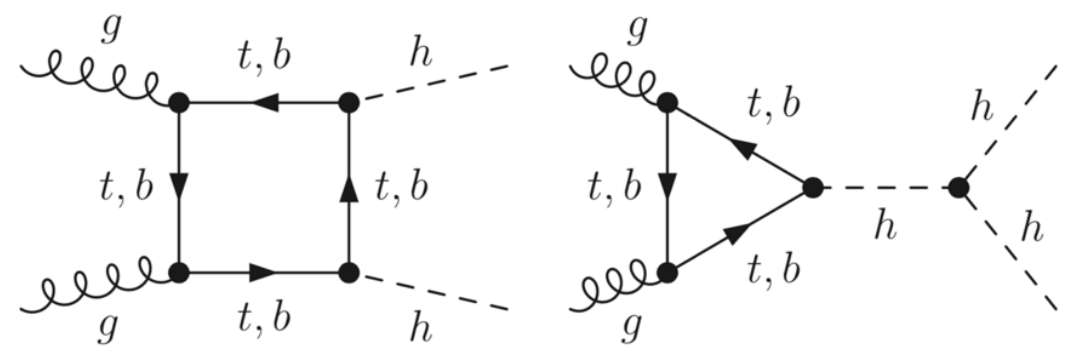

Figure 4: Di-Higgs production processes at the FCC-hh. These two diagrams negatively interfere.

Negative log-Likelihood scans as a function of the trilinear self-coupling modifier $\kappa_{\lambda}=\frac{\lambda_{3}}{\lambda_{S M}}$ are performed for various decay channels and their combination is calculated, see figure 5 . Depending on the assumed detector performance and systematic uncertainties, the Higgs self-coupling could be measured with a precision in the range $3.4-7.8 \%$ at the $68 \%$ CL for an integrated luminosity of $30 \mathrm{ab}^{-1}[10]$.
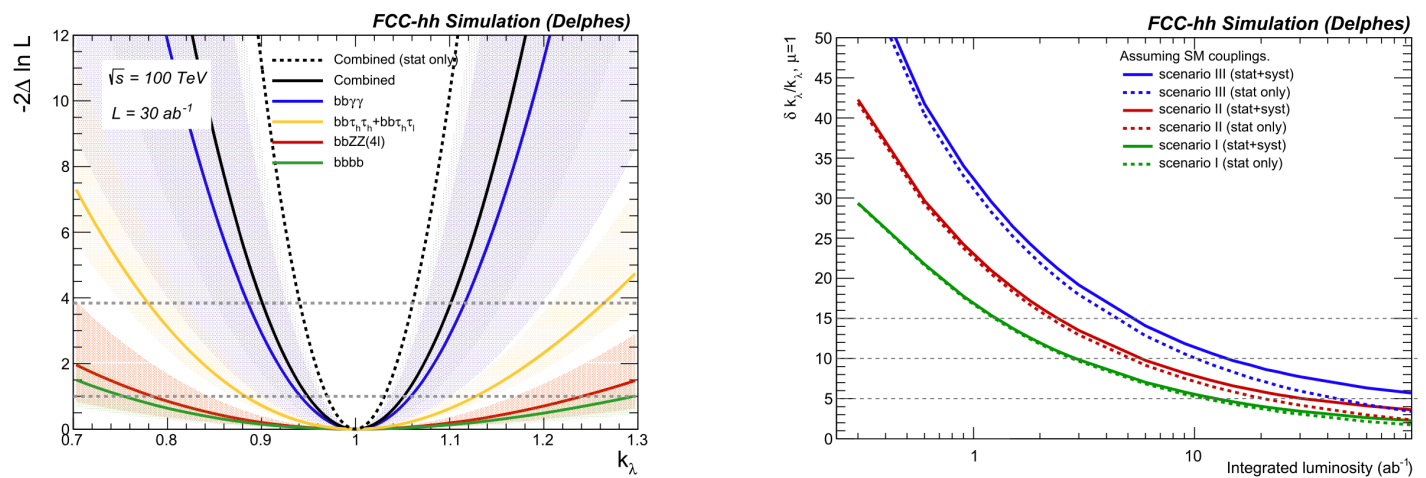

Figure 5: Negative log-Likelihood scans as a function of the trilinear self-coupling modifier $\kappa_{\lambda}=\frac{\lambda_{3}}{\lambda_{S M}}$ (left) and expected precision on the Higgs self-coupling as a function of the integrated luminosity (right) [10]. 


\section{Conclusions}

The large sample of Higgs boson events produced at FCC-ee will allow to measure the HZ cross section with extreme precision and to provide a model-independent measurement of the Higgs boson total width. Most Higgs boson couplings to Standard Model particles will be measured at the sub-percent level. By combining the data from FCC-ee and FCC-hh a precision on the Higgs boson self-coupling of the order of 5\% can be obtained. FCC-ee and FCC-hh will therefore provide the best possible Higgs boson measurements of any new accelerator.

\section{References}

[1] http://hilumilhc.web.cern.ch.

[2] CMS Collaboration, Sensitivity projections for Higgs boson properties measurements at the $H L-L H C$, CMS PAS FTR-18-011, https://cds . cern. ch/record/2647699;

ATLAS Collaboration, Projections for measurements of Higgs boson cross sections, branching ratios, coupling parameters and mass with the ATLAS detector at the HL-LHC, ATL-PHYSPUB-2018-054, https://cds. cern.ch/record/2652762.

[3] https://fcc.web.cern.ch/Pages/default.aspx.

[4] Alain Blondel and Patrick Janot, FCC-ee overview: new opportunities create new challenges, arXiv:2106.13885.

[5] https://fcc.web.cern.ch/Pages/fcc-hh.aspx.

[6] J. de Blas et al., Higgs Boson Studies at Future Particle Colliders, arXiv: 1905. 03764.

[7] M. Selvaggi, DELPHES 3: A modular framework for fast-simulation of generic collider experiments, J. Phys. Conf. Ser. 523 (2014) 012033.

[8] M. Antonello, IDEA: A detector concept for future leptonic colliders, IL NUOVO CIMENTO 43C (2020), DOI $10.1393 /$ ncc/i2020-20027-2.

[9] J. Eysermans, Higgs mass and model-independent cross-section studies from the recoil mass, https://indico.cern.ch/event/995850/contributions/4415989/.

[10] M.L. Mangano, G. Ortona, M. Selvaggi, Measuring the Higgs self-coupling via Higgs-pair production at a $100 \mathrm{TeV}$ pp collider, Eur. Phys. J. C. 80 (2020) 1030, https://doi.org/10.1140/epjc/s10052-020-08595-3. 